\title{
ACOUSTIC INSTABILITY IN COSMIC RAY MEDIATED SHOCKS
}

\author{
HYESUNG KANG AND T. W. JONES \\ School of Physics and Astronomy, University of Minnesota, Minneapolis, MN 55455 \\ AND \\ DONGSU RYU \\ Princeton University Observatory, Peyton Hall, Princeton, NJ 08544 \\ Received 1991 May 24; accepted 1991 August 1
}

\begin{abstract}
We have examined the acoustic instability in cosmic ray dominated media that can amplify sound waves shorter than the scale height of the cosmic-ray pressure. The effects of the instability on the particle distribution have been studied using a time-dependent numerical method in which the diffusion-advection transport equation for the particle distribution function is solved self-consistently with the hydrodynamic conservation equations. Incident sound waves can grow into shocks in the precursors of strong cosmic ray mediated shocks, so that the gas entropy is increased significantly before shock passage, and the postshock cosmic ray pressure is slightly decreased. Fresh particles can be injected from the gas at the small-scale shocks, even after the initial large-scale shock becomes smooth due to the development of a strong cosmic ray pressure precursor. However, even though the instability can amplify the sound waves into moderately strong gas shocks, the cosmic ray pressure and the particle distribution are not significantly affected by these shocks. This is because diffusion of the cosmic rays causes the perturbation on the particle distribution to be much smaller than those of gasdynamic variables.

We suggest the possibility that the amplified small-scale density structures might produce ESE, a class of flux variation observed in some compact radio sources and thought to be caused by scattering or refraction in the intervening interstellar medium.
\end{abstract}

Subject headings: acceleration of particles - cosmic rays: general - instabilities - shock waves

\section{INTRODUCTION}

It is generally believed that the cosmic rays (hereafter CRs) should play important dynamical roles in the interstellar medium (ISM), because the energy density of these high-energy particles is comparable to the thermal energy density of the gas or the magnetic field in the ISM (e.g., Blandford \& Eichler 1987). Ptuskin (1981) examined how sound and MHD waves are damped by the friction due to the diffusion of CRs. For short wavelengths, however, sound waves (Drury 1984; Dorfi \& Drury 1985; Drury \& Falle 1986; hereafter DF), and magnetosonic waves (Chalov 1988; Zank, Axford, \& McKenzie 1990) can be amplified in the presence of a large-scale gradient of CR pressure. These studies have shown that small compressional disturbances propagating through a CR dominated medium can be amplified by an acoustic instability to nonlinear amplitudes if the following two conditions are satisfied: (1) the perturbation wavelength is shorter than the scale height of the CR pressure and (2) the scale height of the CR pressure is smaller than the diffusion length associated with the sound speed. When both conditions are satisfied, the disturbances traveling in the direction of increasing CR pressure will grow. Hereafter we will refer this instability as Drury's instability.

The precursor in CR-dominated shocks of high Mach numbers is always unstable to the growth of sound waves shorter than the scale height of the precursor, because the scale height of the CR pressure gradient in the precursor is several diffusion lengths, $x_{d s}$ (defined as $x_{d s}=\kappa / u_{s}$, where $\kappa$ is the CR diffusion coefficient and $u_{s}$ is the shock velocity), which is always smaller than the diffusion length associated with the sound wave $x_{d c}=\kappa / c_{s}$ (Dorfi \& Drury 1985; DF). The growth of the instability, however, is limited by the fact that the dura- tion of the growth is the time for the wave to be advected through the precursor from upstream to downstream. That is because the wave travels in the direction of strongly increasing $\boldsymbol{P}_{c}$ only in the precursor. Once advected to the postshock region, the amplitudes will remain constant or be damped out, depending on the postshock CR pressure gradient. Dorfi \& Drury (1985) suggested that the sound waves grow into smallscale shocks in the precursor, resulting there in the increase of the gas entropy and the injection of particles from the gas into the CR. In addition, one can expect the particle injection to continue at small-scale shocks even after the total large-scale shock becomes smoothed. Using the two-fluid model DF showed numerically that this instability does occur and that the sound waves become small scale shocks in the precursor of the CR-dominated shocks. Dorfi $(1984,1990)$ also discussed how Drury's instability can grow out of numerical noise in his adaptive grid hydrodynamical code.

In the present paper, we attempt to provide a clear understanding of the underlying physics of this instability. We have reexamined the linear stability analysis previously done by DF using the two-fluid model. In addition, using both two-fluid and diffusion-advection equation numerical approaches, we have explored the nonlinear development of the instability. In the two-fluid model (Drury \& Völk 1981; Kang \& Jones 1990; Jones \& Kang 1990) CRs are treated as a second, massless fluid described by a pressure and a specific heat ratio. The CR energy equation with a mean (momentum averaged) diffusion coefficient is solved instead of the diffusion-advection transport equation for the particle distribution function (see Jones \& Kang 1990 for a detailed description of our two-fluid code). A more detailed view of the behavior of the instability is afforded 
with our transport code using the numerical methods described in Kang \& Jones (1991 hereafter KJ). There the diffusionadvection transport equation for the particle momentum distribution function is solved directly. Solving the transport equation eliminates the necessity of assuming values for the momentum averaged diffusion coefficient and the ratio of specific heats for the CRs. In addition this enables us to ask several interesting questions which would have not been possible with the two-fluid model. First, we can see how smallscale shocks will affect the particle distribution for a diffusion coefficient dependent on the particle momentum. One might expect that the acceleration to the particle momenta corresponding to the velocities of small-scale shocks may take place faster in the presence of the instability than in the unperturbed medium. Second, the effects of in situ CR injection from the thermal plasma at small-scale shocks can be studied. If the particles are injected at shocks according to the model in which the injection momentum is proportional to the postshock thermal velocity (Falle \& Giddings 1987; Jones \& Kang 1990; $\mathrm{KJ}$ ), the injection will take place with lower energies in smallscale shocks. Dorfi \& Drury (1985) suggested that the increase of the particle numbers with low momenta may effectively decrease the mean diffusion coefficient, reducing the mean accleration time scale, so that higher particle energies could be reached. One goal of this work is to study how significantly the instability may change the particle distribution and the CR energy density due to the small scale shocks and the associated particle injection on the precursors of strong CR mediated shocks. On the other hand, one can expect that the diffusive smoothing of the CR fluctuations will work to minimize that effect, even though diffusion is the physical basis for the existence of the instability in first place.

\section{BASIC EQUATIONS}

The governing equations for cosmic rays and the thermal plasma can be found in numerous studies, some of which are referenced in the previous section. We will use the same equations and variables as in KJ. Assuming strong scattering of cosmic rays, we solve the transport equation for the isotropic part of the distribution function according to

$$
\frac{d f}{d t}=\frac{1}{3}(\nabla \cdot u) p \frac{\partial f}{\partial p}+\nabla \cdot(\kappa \nabla f)+Q
$$

where $f(x, p, t)$ is the number density of particles in phase space, $d / d t$ is the total time derivative, $\kappa$ is the diffusion coefficient (most generally a tensor, but considered a scalar in the present paper), and $\boldsymbol{u}$ is the background fluid velocity. The source term $Q$ represents particle injection at the gas subshock (see eq. [2.8] in $\mathrm{KJ}$ ).

In the two-fluid model equation (2.1) is multiplied by the kinetic energy of particles and integrated with respect to momentum. This produces the energy conservation equation for the CR,

$$
\frac{d E_{c}}{d t}=-\gamma_{c} E_{c}(\nabla \cdot u)+\nabla \cdot\left(\langle\kappa\rangle \nabla E_{c}\right)+S
$$

where $E_{c}$ and $\gamma_{c}$ are the CR energy density and the ratio of specific heats, respectively, and $S$ represents the energy transfer rate from the gas to $\mathrm{CR}$ due to the particle injection. (see eqs.
[2.5]-[2.9] in $\mathrm{KJ})$. Here the mean diffusion coefficent is defined according to

$$
\langle\kappa\rangle=\frac{\int_{p_{0}}^{p_{1}}(\partial f / \partial x) \kappa\left[\sqrt{p^{2}+1}-1\right] p^{2} d p}{\int_{p_{0}}^{p_{1}}(\partial f / \partial x)\left[\sqrt{p^{2}+1}-1\right] p^{2} d p},
$$

where the momentum is expressed in units of $m c$.

The background fluid follows the equations of ideal gas dynamics generalized to include CR pressure as follows,

$$
\begin{gathered}
\frac{d \rho}{d t}=\rho \nabla \cdot u, \\
\frac{d \boldsymbol{u}}{d t}=-\frac{1}{\rho} \nabla\left(P_{g}+P_{c}\right), \\
\frac{d e}{d t}=-\frac{1}{\rho} \nabla \cdot\left\{\left(P_{g}+P_{c}\right) u\right\}+\frac{1}{\rho} P_{c} \nabla \cdot u-\frac{S}{\rho} .
\end{gathered}
$$

Here, $P_{g}$ and $P_{c}$ are the pressure contributed from gas and CR particles, respectively, while $e$ is the sum of gas thermal and kinetic energy per unit mass.

Equations (2.1) and (2.2) are solved using the implicit, Crank-Nicholson scheme. The gas dynamic equations are solved using the Piecewise Parabolic Method (PPM) hydrodynamic scheme (Colella \& Woodward 1984), including the added terms necessary to treat CR pressure (see Appendix A). In the present paper, which deals solely with one spatial dimension, the numerical calculations in which equation (2.1) is solved will be called transport calculations, while those using equation (2.2) assuming specific values for $\gamma_{c}$ and $\langle\kappa\rangle$ will be called two-fluid calculations.

\section{PHYSICAL DESCRIPTION}

Perturbations in a CR mediated gas flow can become unstable because the CR pressure may not be perturbed adiabatically due to rapid diffusion. In the limit of infinitely rapid diffusion, CRs are completely decoupled from the gas for small-scale perturbations and a large scale gradient of $\mathrm{CR}$ pressure will remain constant. In this limit, the CR pressure will exert an approximately constant volume force to the gas and the growth rate of the instability will maximize. To see this, suppose a constant volume force, $F$, is exerted on the gas so that the acceleration is $F / \rho$. Then, a compressed region in a wave train will be accelerated in the opposite direction of the force, while a decompressed region will be accelerated in the direction of the force (i.e., $\delta$ acceleration $=-F \delta \rho / \rho^{2}$ ). As a result, oscillating density disturbances (or sound waves) moving opposite to the direction of the force will suffer an extra restoring force and their amplitude will grow. Likewise, waves propagating in the other direction will be damped. This explains why disturbances traveling in the direction of the increasing CR pressure will be amplified, while those traveling in the opposite direction will be decaying. On the other hand, if the diffusion is finite, the damping of CR pressure due to diffusive "bulk" viscosity will effectively provide a friction against traveling disturbances. This is the damping effect first identified by Ptuskin (1981).

The existence of the instability is established by two competing forces; the CR pressure gradient and Ptuskin's damping. The former should dominate over the latter in order for the instability to occur. Hence, the conditions for the insta- 
bility, which were originally derived by DF using the waveaction equation in the two-fluid model, are

$$
\frac{1}{k}<\frac{P_{c}}{\left|\nabla P_{c}\right|}<\frac{|(1+\beta)|\langle\kappa\rangle}{\gamma_{c} c_{s}} .
$$

Here, $k$ is the wavenumber of the perturbations and $\beta=(\delta \kappa) / \kappa /$ $(\delta \rho / \rho)$. We can define the instability number, $D$,

$$
D=\frac{\langle\kappa\rangle\left|(1+\beta) \nabla P_{c}\right|}{\gamma_{c} c_{s} P_{c}}
$$

In the regions where $D>1$, small compressional disturbances will be amplified due to the Drury's instability.

\section{LINEAR ANALYSIS}

Although the detailed discussion of the linear stability analysis can be found in DF, we reexamine the simplest case involving an isothermal, exponential background in order to clarify some characteristics to be used later. With the exponential background defined as

$$
\begin{aligned}
\bar{\rho} & =\rho_{0} \exp \left(\frac{x}{L}\right), \\
\bar{P}_{g} & =P_{g 0} \exp \left(\frac{x}{L}\right), \\
\bar{P}_{c} & =P_{c 0} \exp \left(\frac{x}{L}\right), \\
\bar{u} & =0, \\
\bar{\kappa} & =\kappa_{0},
\end{aligned}
$$

let us consider the linear perturbations of the form

$$
\begin{aligned}
\tilde{\rho} & =\rho_{1} \exp \left(\frac{x}{L}+i w t-i k x\right), \\
\tilde{P}_{g} & =P_{g 1} \exp \left(\frac{x}{L}+i w t-i k x\right), \\
\tilde{P}_{c} & =P_{c 1} \exp \left(\frac{x}{L}+i w t-i k x\right), \\
\tilde{u} & =u_{1} \exp (i w t-i k x), \\
\tilde{\kappa} & =\beta \frac{\rho_{1} \kappa_{0}}{\rho_{0}} \exp (i w t-i k x) .
\end{aligned}
$$

For $k \gg 1 / L \gtrsim c_{s} / \kappa_{0}$, the linearized perturbation equations become

$$
\begin{gathered}
w \frac{\rho_{1}}{\rho_{0}}-k u_{1}=0 \\
i w u_{1}-\frac{1}{L} \frac{P_{g 0}}{\rho_{0}} \frac{\rho_{1}}{\rho_{0}}-\frac{1}{L} \frac{P_{c 0}}{\rho_{0}} \frac{\rho_{1}}{\rho_{0}}-i k \frac{P_{g 1}}{\rho_{0}}-i k \frac{P_{c 1}}{\rho_{0}}=0 \\
w \frac{P_{g 1}}{P_{g 0}}-\gamma_{g} k u_{1}=0 \\
k \kappa_{0} \frac{P_{c 1}}{P_{c 0}}+\frac{i \beta_{\kappa 0}}{L} \frac{\rho_{1}}{\rho_{0}}-i \gamma_{c} u_{1}=0 .
\end{gathered}
$$

With $w=a-b i$ and $a \sim c_{s} k \gg b$ (see DF), equations (4.3a) and (4.3c) indicate that $\rho_{1} / \rho_{0}$ and $P_{g 1} / P_{g 0}$ are the same order as $u_{1} / c_{s}$. On the other hand, equations (4.3a) and (4.3d) indicate that for $\beta=0, P_{c 1} / P_{c 0} \sim u_{1} /\left(k \kappa_{0}\right) \ll u_{1} / c_{s}$. This demonstrates that because of CR diffusion the amplitude of $\widetilde{P}_{c}$ is much smaller than that of other gas dynamic variables.

By combining equations (4.3b) and (4.3d), we get

$$
i w u_{1}-\frac{1}{L} \frac{P_{g 0}}{\rho_{0}} \frac{\rho_{1}}{\rho_{0}}-i k \frac{P_{g 1}}{\rho_{0}}+\frac{\gamma_{c}}{\kappa_{0}} \frac{P_{c 0}}{\rho_{0}} u_{1}-\frac{\beta+1}{L} \frac{P_{c 0}}{\rho_{0}} \frac{\rho_{1}}{\rho_{0}}=0 \text {. }
$$

While the second and third terms describe sound waves which are neutrally oscillating, the fourth and last terms cause the sound wave to decay or grow. Here, the fourth term is Ptuskin's damping term due to the CR diffusion. The last term is the term which makes the sound waves grow. It is clear from this term that, if $\beta=-1$, so that $\tilde{\kappa} / \bar{\kappa}=-\tilde{\rho} / \bar{\rho}$, the force driving the instability disappears, and the instability does not occur.

In the limit with very large CR pressure, or $P_{c 0} \gg P_{g 0}$ and $P_{c 0} \gg \kappa_{0} \rho_{0} c_{s} k$, the terms involving gas pressure and gas pressure perturbation in the perturbed momentum equation ( $4.3 \mathrm{~b})$ become negligible. Then, the waves are no longer driven by gas pressure, but by CR pressure or diffusion. It turns out that the waves driven by $\mathrm{CR}$ pressure are a decaying mode, giving approximately

$$
w \sim \pm \sqrt{\frac{\gamma_{c} P_{c 0}}{\rho_{0}}} k+\frac{i}{2} \kappa_{0} k^{2}
$$

while those driven by diffusion are a growing mode, giving approximately

$$
w \sim \frac{\kappa_{0}}{L \gamma_{c}} k-i \frac{\kappa_{0}^{3}}{L^{2} \gamma_{c}^{3}} \frac{\rho_{0}}{P_{c 0}} k^{2} .
$$

Hence, in this limit the growth rate, $b$, is inversely proportional to $\mathrm{CR}$ pressure.

The full dispersion relation for the case with an isothermal, exponential background is given in DF. We have calculated $w$ from that dispersion relation to study the growth rates in several different limits. As pointed out by DF one should distinguish between "adiabatic" changes in the wave amplitudes induced by variations in the background and "genuine" growth due to instabilities. The instability condition (3.1), which is derived using the wave-action equation in the shortwavelength limit, is required for "genuine" growth. On the other hand, the imaginary part of $w$ contains both "adiabatic" changes and "genuine" growth, because we define the perturbation equations as in equations (4.2). [If we replace the factor $\exp (x / L)$ with $\exp \{x /(2 L)\}$ in equations (4.2), then the imaginary part of $w$ will represent only "genuine" growth.] For instance, $w= \pm c_{s} k+(i / 2)\left\{\gamma_{c} P_{c 0} /\left(\rho_{0} \kappa_{0}\right) \mp P_{c 0}(1+\beta) /\left(L \rho_{0} c_{s}\right) \pm\right.$ $\left.c_{s} / L\right\}$ in the short-wavelength limit (DF). Here the term $c_{s} / L$ represents the adiabatic changes due to the exponential background. The growth rate, $b$, is plotted in Figures $1 a-1 d$ for the case of $L=1, \rho_{0}=1, \gamma_{c}=5 / 3$, and for wide ranges of $\kappa_{0}, P_{c 0}$, $P_{g 0}$, and $\lambda$. From the instability criterion (3.1), $P_{g 0}$ should be less than 0.216 for $P_{c 0}=1, \gamma_{c}=5 / 3, \kappa_{0}=1$, and $\beta=0$ in order that there exits a "genuinely" growing mode, while $\boldsymbol{P}_{g 0}$ should be less than 0.13 in order that $w$ has the nonzero imaginary part (see Fig. 1b). According to Figure $1 a$ the growth rate is relatively insensitive to the wavelength of the perturbations and is largest in the limit of small wavelength. Figures $1 b$ and $1 c$ show that a maximum growth rate is achieved in the limit of cold gas or in the limit of infinite diffusion, as mentioned in the 

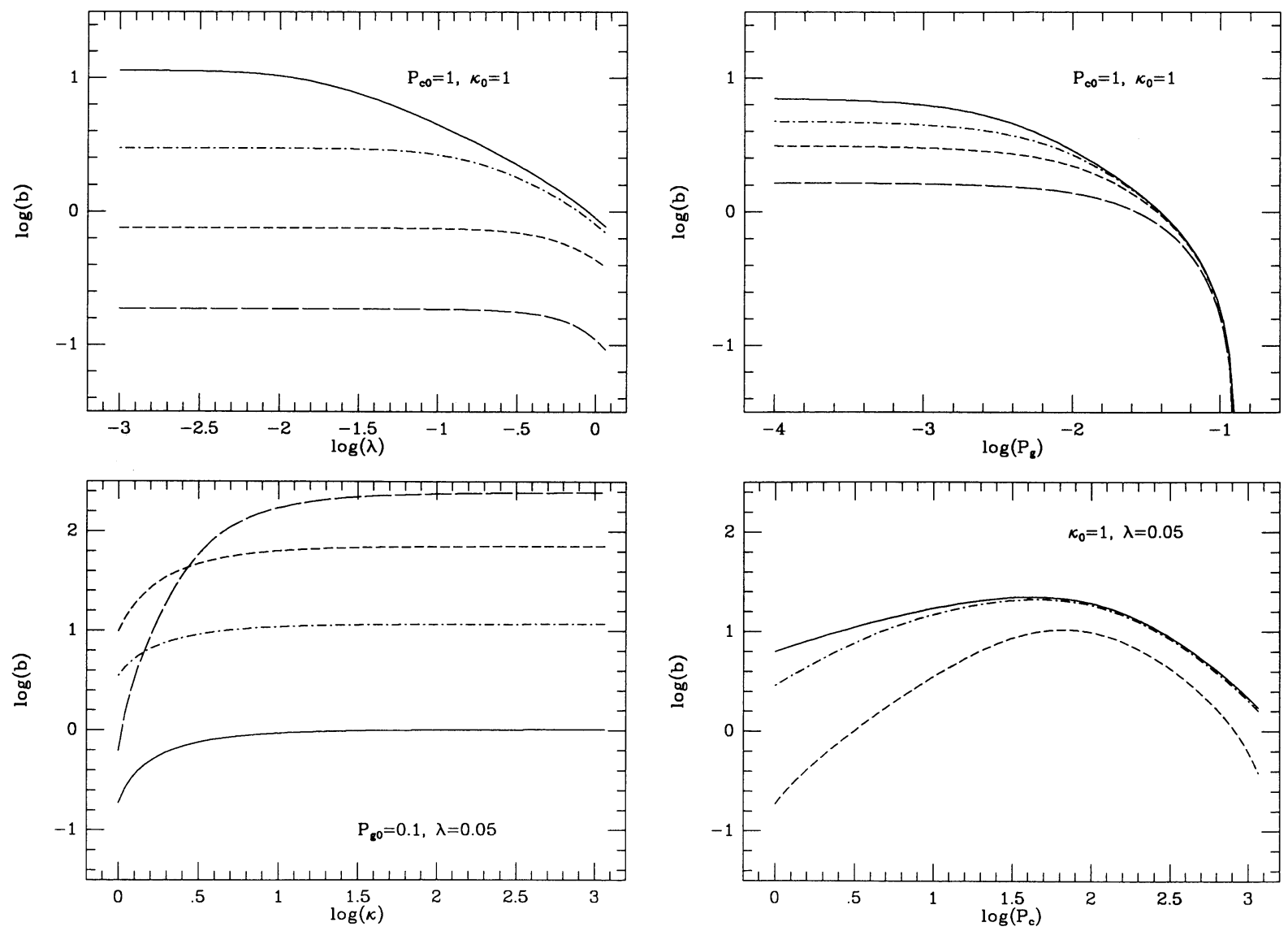

FIG. 1.-Growth rates of sound waves in the exponential background for $L=1, \rho_{0}=1, \gamma_{c}=5 / 3$. Dependence is shown against various parameters. (a) $P_{c 0}=1$, $\kappa_{0}=1$, and $P_{o 0}=0.0001$ (solid line), 0.01 (dot-dashed line), 0.05 (dashed line), and 0.1 (long-dashed line). (b) $P_{c 0}=1, \kappa_{0}=1$, and $\lambda=0.05$ (solid line), 0.1 (dot-dashed line), 0.2 (dashed line), and 0.5 (long-dashed line). (c) $P_{g 0}=0.1, \lambda=0.05$, and $P_{c 0}=1$ (solid line), 10 (dot-dashed line), 100 (dashed line), and 1000 (long-dashed line). (d) $\kappa_{0}=1, \lambda=0.05, P_{g 0}=0.001$ (solid line), 0.01 (dot-dashed line), 0.1 (dashed line).

previous section. Finally, Figure $1 d$ exhibits the decrease of the growth rate as $\mathrm{CR}$ pressure becomes infinite, because of increased Ptuskin damping.

\section{NUMERICAL RESULTS}

\subsection{Two-Fluid Calculations}

We have calculated the evolution of piston driven $C R$ shocks using the two-fluid model with $\rho=1, P_{g}=10^{-3}$, and a preexisting CR pressure, $P_{c}=10^{-3}$ far upstream. The piston velocity, $u_{p}=1$. The assumed values of the specific heats and the mean diffusion coefficient are $\gamma_{c}=1.6$ and $\langle\kappa\rangle=1(\beta=0)$, respectively. The Mach number of the shock driven by this piston is about 33. The calculation is done in the frame where the piston is at rest, so beginning at $t=0$, the uniform, incoming flow is moving with $u=-1$ toward the piston standing at the left boundary. The sound waves are generated at the right boundary with the velocity perturbation, $u=A c_{s 0} \sin (w t)$, where $A$ and $c_{s 0}$ are the normalized amplitude and unperturbed sound speed, respectively. The Doppler shifted wave frequency, $w=(2 \pi / \lambda)\left(u_{p}+c_{s 0}\right)$. The density and gas pressure at the right boundary are adiabatically perturbed so that the Riemann invariant, $\boldsymbol{R}_{+}=u+2 C_{s} /\left(\gamma_{g}-1\right)=$ constant (i.e., a sound wave moving to the left), where $C_{s}$ is the Lagrangian sound speed.
In CR modified shocks, the amplitude of the sound wave coming from upstream grows while the wave is advected downstream through the precursor. After the wave climbs up to the peak of CR pressure, it tends to be damped out in the postshock region where CR pressure decreases. Therefore, the growth time of the instability acting on an individual wave is limited to the time during which the sound wave is advected downstream through the precursor. This growth time is several shock diffusion times $\left(t_{d s}=\kappa / u_{s}^{2}\right)$. In the present simulation the diffusion time scale, $t_{d s} \sim 1$. On the other hand, the postshock region sometimes can be unstable for sound waves propagating from postshock region to the preshock region if the conditions given in equation (3.1) are satisfied.

Figure 2 shows the density, gas pressure, gas entropy and the CR pressure, and the velocity at $t=100$ for a wave with $\lambda=2$. The instability number $D$ in equation (3.2) is plotted for the unperturbed case. $D$ is larger than unity in the precursor region $(29<x<39)$ and peaks at $x=34$. The waves are amplified inside the precursor and become small-scale shocks, resulting in the increase of the gas entropy inside the precursor. Even though the gas pressure increases at small scale shocks compared to the background, the CR pressure shows an almost smooth structure, consistent with what we found in $\S 4$. For small amplitudes, $A \lesssim 0.01$, the advection time across the precursor is not long enough for the waves to grow into shocks in 

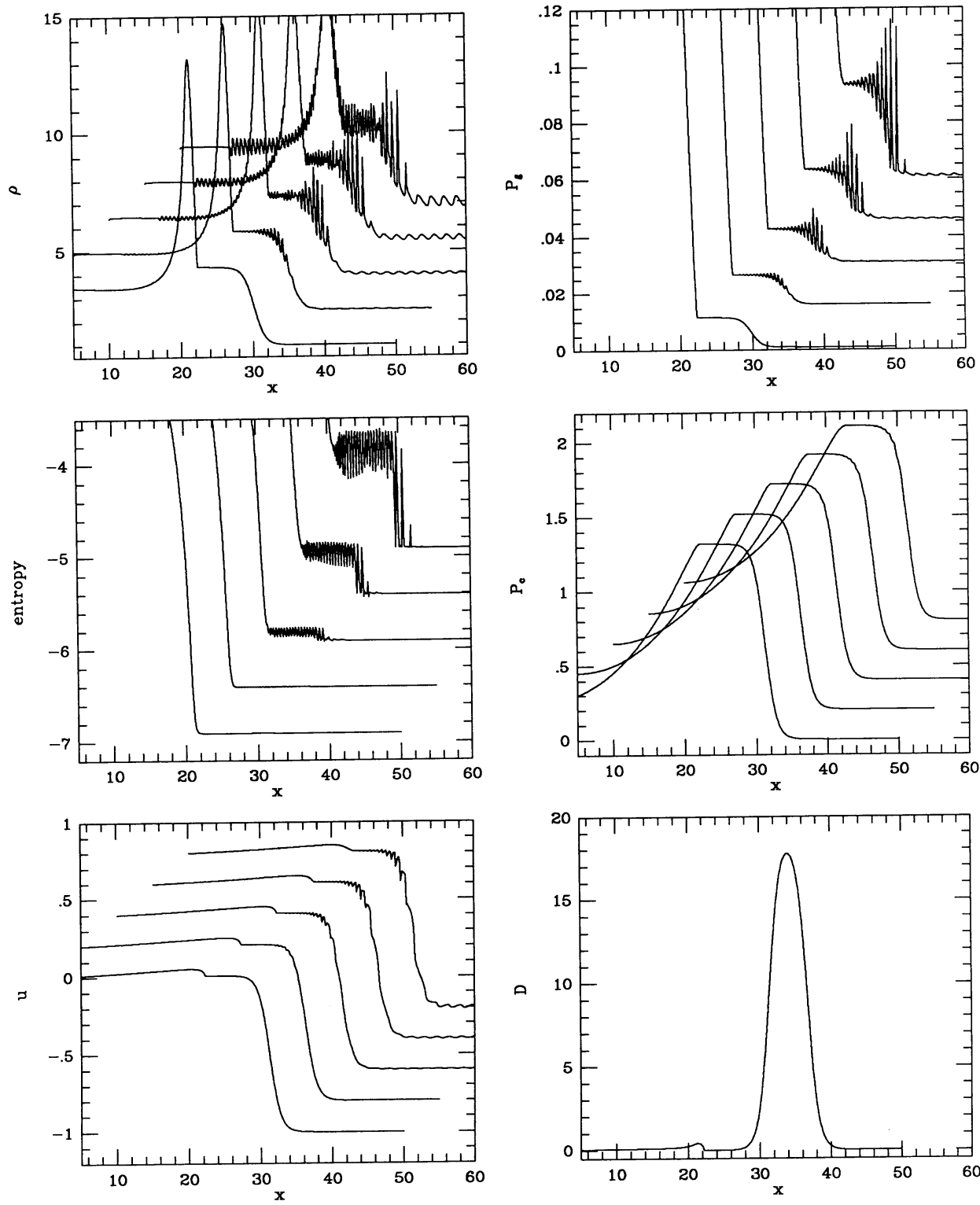

FIG. 2-Piston-driven cosmic-ray shocks $\left(u_{p}=1\right)$ at $t=100$, propagating into a medium with $\rho=1, P_{g}=0.001, P_{c}=0.001, \kappa=1$, and $\gamma_{c}=1.6$. Shocks have been perturbed by sound waves of wavelength $\lambda=2$ and amplitudes $A=0,0.01,0.05,0.1,0.2$ (from bottom left to top right). Plots for perturbed cases are shifted diagonally for the clarity. The instability number $D$ is plotted for the unperturbed case $(A=0)$.

this particular model. For large initial amplitudes, $A \gtrsim 0.05$, the small-scale shocks form and the entropy increase due to these shocks is roughly proportional to the initial amplitude. The advection time scale will increase linearly with the diffusion coefficient, because the scale height of the precursor is the order of the diffusion length of the shock $\left(\kappa / u_{s}\right)$. Also the linear growth rate increases with the diffusion coefficient as discussed in $\S 4$. Hence the amplification of the waves will be greater for larger diffusion coefficients as long as the lifetime of the shock is long enough to reach a CR-mediated state.

We found that the incoming waves grow only adiabatically in the simulations with the diffusion coefficient inversely proportional to the density (i.e., $\beta=-1$ ). This is consistent with the linear growth analysis that Drury's instability does not work for $\beta=-1$.

\subsection{Transport Calculations}

We have also considered piston driven CR shocks similar to the ones in the previous section using the transport code. For these calculations we studied shocks in flows containing preexisting CR as before, and, in addition, shocks with no preexisting CR, but in which fresh, low-energy CR can be injected in situ at a shock. The initial particle distribution function for the cases of prexisting particles is proportional to $p^{-5}$ from $p_{0}=$ $4.5 \times 10^{-5}$ to $p_{1}=2.2 \times 10^{4}$ ( $p$ is the particle momentum in units of $m c$ ). It is normalized so that the initial, uniform $\boldsymbol{P}_{c}=$ $10^{-3}$. We also calculated cases in which particles are injected at shocks according to the model described in $\mathrm{KJ}$ in which a small fraction, $\epsilon$, of the incoming thermal particles are injected with a momentum corresponding to twice the postshock sound 
TABLE 1

\begin{tabular}{clccr}
\multicolumn{5}{c}{ MODELS } \\
\hline \multicolumn{1}{c}{ Model } & \multicolumn{1}{c}{$\alpha$} & \multicolumn{1}{c}{$P_{c}$} & \multicolumn{1}{c}{$\epsilon$} & \multicolumn{1}{c}{$\Delta x$} \\
\hline P1 $\ldots \ldots \ldots \ldots$ & 0 & $10^{-3}$ & 0 & $5 \times 10^{-2}$ \\
P2 $\ldots \ldots \ldots \ldots$. & 0.25 & $10^{-3}$ & 0 & $2.5 \times 10^{-2}$ \\
P3 $\ldots \ldots \ldots \ldots$ & 0.5 & $10^{-3}$ & 0 & $1.7 \times 10^{-2}$ \\
I1 $\ldots \ldots \ldots \ldots \ldots$ & 0 & 0 & $10^{-2}$ & $5 \times 10^{-2}$ \\
I2 $\ldots \ldots \ldots \ldots \ldots$ & 0.25 & 0 & $10^{-2}$ & $2.5 \times 10^{-2}$ \\
I3 $\ldots \ldots \ldots \ldots$. & 0.5 & 0 & $10^{-2}$ & $1.7 \times 10^{-2}$ \\
\hline
\end{tabular}

speed. In the present work we allowed the injection process at the shock whose Mach number is greater than 2. We believe details of the injection model should not affect the general characteristics of our results.

The model parameters are summarized in Table 1. The numerical resolution in logarithmic momentum space is $\Delta(\ln p)=0.156$. The ratio of the piston velocity to the speed of light is $10^{-2}$. This choice makes the shock velocity and the injected particles nonrelativistic, so the $\gamma_{c}$ is initially close to $5 / 3$. With the same initial $P_{c}$ a smaller piston velocity wouldn't change much except to make it take longer for particles to become relativistic. A larger value would make the shock and the injected particles almost relativistic, so that $\gamma_{c}$ is initially close to $4 / 3$. This will make the dynamic evolution much slower than the case with initial $\gamma_{c} \sim 5 / 3$ (Jones \& Kang 1990).

We have included a diffusion coefficient dependent on the particle momentum, $\kappa(p)=p^{\alpha}$, where $\alpha=0,0.25$, and 0.5 . One should notice that the momentum dependent diffusion coefficient has a wide dynamic range for the particle momenta included in our calculation. With currently available computing resources, it is not possible for us to achieve numerical convergence for the lowest energy particles with $p \ll 1$ for a diffusion coefficient even weakly dependent on momentum, since the resolution of the spatial grid required for an adequate convergence is $\Delta x \approx 0.05 \kappa(p) / u$. For the high-energy particles with $p \gg 1$ the diffusion length becomes longer than the length of our numerical grid when $\alpha>0$, so the high energy particles cannot be followed properly. These particles will be effectively trapped inside the numerical grid, since we are using the boundary condition in which no diffusive flux is allowed across the boundaries. Because of these practical difficulties we did not include the case of $\alpha=1$ or 2 which could be physically more realistic in some models. For the simulations with a momentum dependent diffusion coefficient, we have done a few calculations with several different spatial resolutions to assure that the general characteristics of the solutions are converged. Each calculation with the highest resolution $\left(\Delta x=1.2 \times 10^{-2}\right)$ took about 12.5 CPU hours on the Cray X-MP supercomputer at Minnesota Supercomputer Institute, while each lowest resolution $\left(\Delta x=5 \times 10^{-2}\right)$ calculation required about 0.7 CPU hours.

Figure 3 and Figure 4 show the shocks driven into both unperturbed and perturbed upstream gas at $t=150$ for the cases of the preexisting particles (model P3) and the injected particles (model I3), respectively. The diffusion coefficients are proportional to $p^{0.5}$. One should be careful in making detailed comparison between perturbed and unperturbed cases, because differences in effective gas thermodynamics cause the shocks in each case to be in a somewhat different position. In general the basic characteristics established from the two-fluid study are confirmed. Unlike two-fluid calculations, however, the shock structure does not reach the steady state, because $\gamma_{c}$ near the shock changes with time. Because the high-energy particles travel further upstream due to the momentum dependent diffusion coefficient, $\gamma_{c}$ decreases upstream as the shock ages. The decrease in $\gamma_{c}$ in the precursor region causes the postshock $\boldsymbol{P}_{c}$ to reach a maximum and then decrease slightly with time. The instability heats the gas inside the precursor, so that the postshock $P_{c}$ is smaller compared to the unperturbed case. This effect is relatively small, but becomes more significant as the dependence of the diffusion coefficient on the particle momentum gets stronger. The decrease in the total CR energy, for example, is only $1 \%$ for model P3 and $7 \%$ for model I3. Analogous reductions in $P_{c}$ have been cited as a consequence of Alfvén wave dissipation and associated heating in the precursor (Völk, Drury, \& McKenzie 1984).

We also visually examined false color images of the distribution function in phase space. For constant diffusion coefficients, the instability has almost no effect on the distribution function in the case of preexisting particles without injection. On the other hand, it causes additional particles to be injected at the low momentum end for the injection cases. For the momentum dependent diffusion coefficient the distribution function is affected in several ways by the instability. In the perturbed cases particles around the highest energy do not travel as far upstream as in the unperturbed cases, so the distribution at $p \sim 10^{4}$ is less affected by the finite grid. One can understand this as due to the "effective" diffusion length $(\kappa /\langle u\rangle)$ for these high-energy particles being decreased by an increase of the mean "effective," or "accumulated" velocity jump $\langle u\rangle$ in the perturbed cases. Each of these small shocks compresses the CRs, requiring them to diffuse all over again into the upstream region. That process will be more important for high momentum particles just because they reside longer in the precursor.

For the diffusion coefficient with strongest momentum dependence the diffusion lengths of the low-energy particles are smaller than the mean diffusion length, and so the low-energy particles do not diffuse much on the scale of the perturbation. As a result, the computed distribution function at the lowenergy end is not as smooth as at higher energies. Figure 5 shows the spatial structure of the distribution function $g=f p^{4}$ at $p=7.3 \times 10^{-3}$ for the momentum-dependent diffusion coefficients (models P2, P3, I2, and I3). The enhancement of particle numbers in the perturbed cases (solid lines) over the unperturbed cases (dashed lines) is obvious. As the momentum dependence of the diffusion coefficient gets stronger, the lowenergy particles (i.e., those with slower diffusion) show more spatial perturbations. For higher energies particle distributions are smooth.

As another means to compare the particle distribution functions we have calculated a "total" distribution function by integrating over the spatial grid, $G(p)=\int f p^{4} d x$. Figure 6 shows the time evolution of $G$ for models P3 and I3. The quantity $G$ at the low-energy end is increasing with time mostly due to the particles flowing into the grid with $f \propto p^{-5}$ (in model P3) or to the particles freshly injected at low momenta (in model I3). The enhancement of particles around $p \sim 10^{-3.5}$ in the perturbed case over the unperturbed case becomes greater with time as the small-scale shocks develop fully and more compression by these shocks takes place. As the acceleration proceeds, the distribution function $f$ becomes proportional to $p^{-4}$ and it becomes steady to higher energy. By the time $t=150$, for example, the slope becomes -4 and the distribution function becomes steady for $10^{-1}<p<10$. The particles 

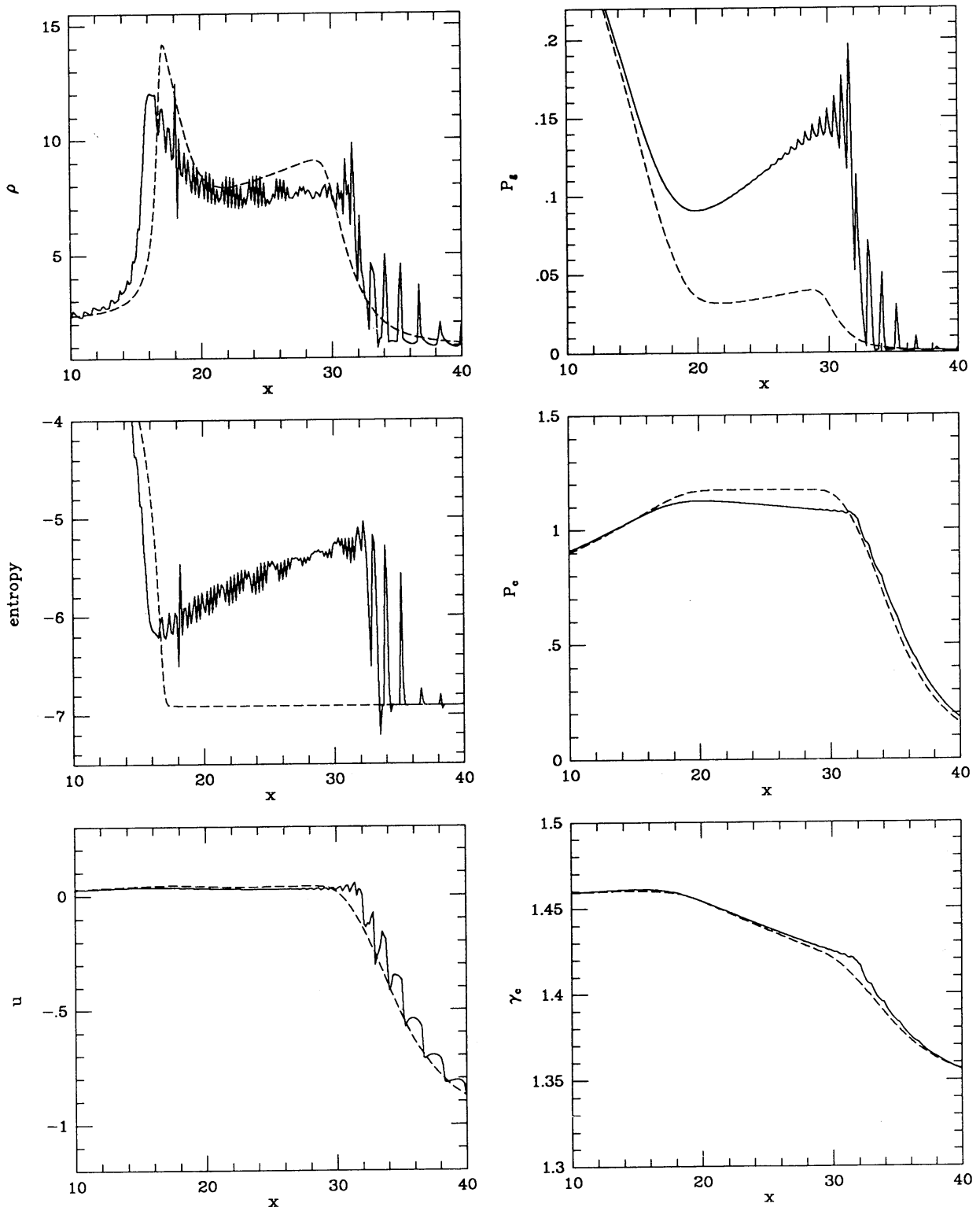

FIG. 3.-Piston driven cosmic-ray shocks $\left(u_{p}=1\right.$ ) at $t=150$, propagating into a medium with $\rho=1, P_{g}=0.001, P_{c}=0.001$, and $\kappa=p^{0.5}$ (model P3). The solid lines are the case perturbed by sound waves of $\lambda=0.2$ and $A=0.2$, while the dashed lines are the unperturbed case. Beating between incoming waves and those reflected off the piston is clearly evident in the density and entropy plots.

flowing into the grid or freshly injected at the small-scale shocks are added to the shock in a time scale shorter than the acceleration time scale, so they tend to pile up around $10^{-3.5}$ This can be seen if we compare the unperturbed and perturbed cases in the injection model I3.

Figure 7 shows $G$ at $t=150$ for both unperturbed and perturbed cases for all the models we include. For the case of preexisting particles, the particle distribution function is not significantly affected by the instabilities in low energies if we assume a constant diffusion coefficient. For the momentum dependent diffusion cases, particles are more efficiently accelerated by small scale shocks to a momentum corresponding to the shock speed (e.g., $p \sim 10^{-3.5}$ ). For the case of injected particles, the particle numbers around $p=10^{-3.5}$ are greatly enhanced due to the injection at small-scale shocks. But the total energy density of the CR is mostly produced by those particle with $1<p<10^{2}$ and the particle numbers in this momentum range are reduced slightly in the perturbed flows for both the preexisting particle models and injection models.

At higher momenta, $10^{2}<p<10^{3}$, there seem to be more particles in the perturbed flows in both the preexisting CR models and the injected CR models. This must be a result of a decrease in the acceleration time scale to these momenta. In order to see if this shorter acceleration time results from the smaller mean diffusion coefficient as Dorfi \& Drury (1985) suggested (see introduction), we have calculated a spatially averaged mean diffusion coefficient, $K$, by numerically integrating $\langle\kappa\rangle$ in the equation (2.3) over the spatial grid using the numerical particle distribution. The quantity $K$ is plotted in Figure 8 against time for $\alpha=0.25$ and 0.5 and for both preexisting particle cases and pure injection cases. In fact, for preexisting particle models the values of $K$ for the perturbed case are 

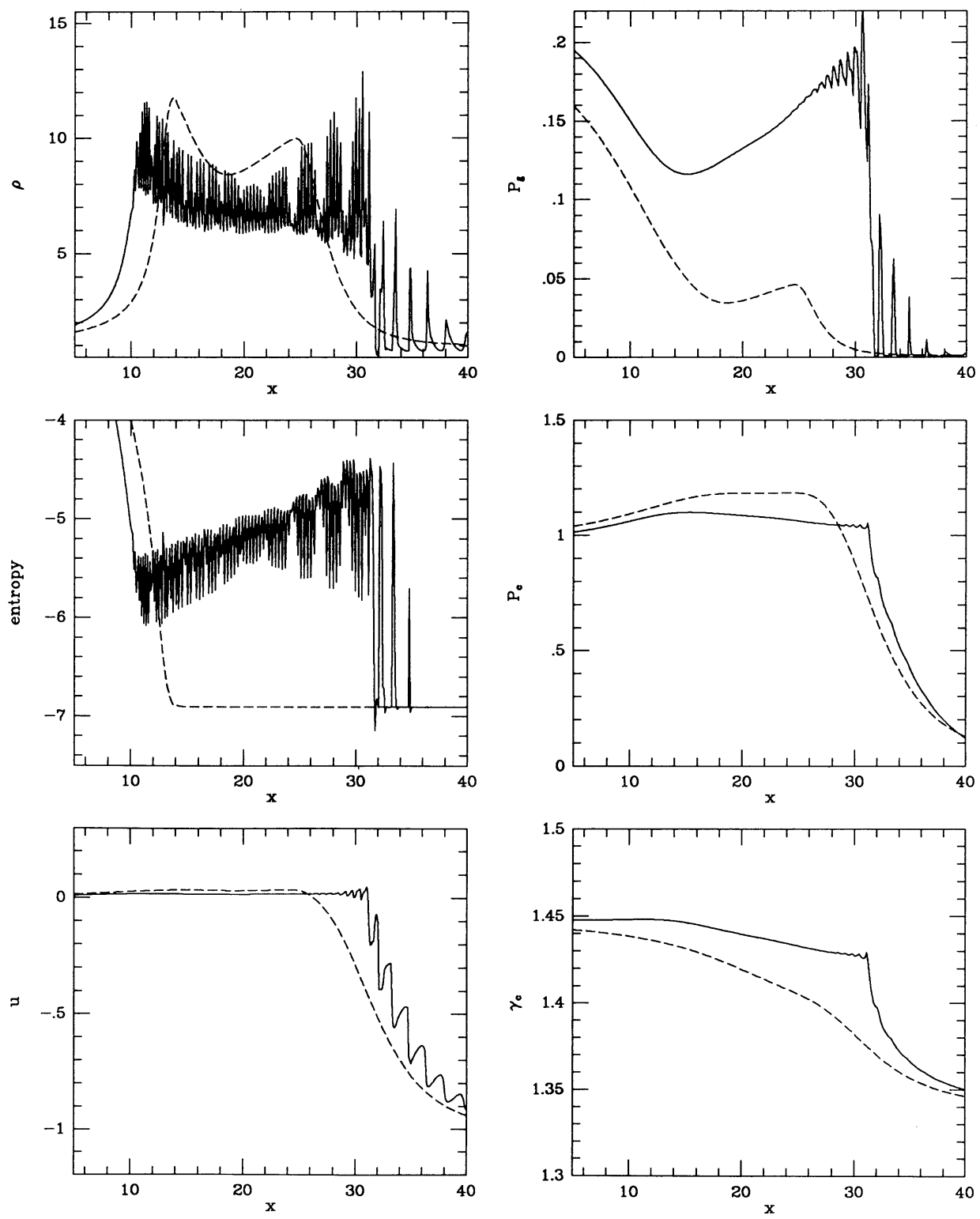

FIG. 4. - Same as Fig. 3 except $P_{c}=0$, and injection parameter $\epsilon=0.01$ (model I3)

a little larger than those for the unperturbed case. This is because of the enhanced particle numbers in the range $10^{2}<p<10^{3}$. For pure injection models, this effect is reduced by the enhancement of the low-energy particles for $p<10^{-1}$, which decreases the mean diffusion coefficient. For the case with $\kappa=p^{0.5}$ the increasing trend is reversed at $t>120$, so that $K$ is smaller in the perturbed case. This is because the influence of injected low-energy particles is greater than that of the excess in high-energy particles mentioned above. The maximum reduction in this case at $t=150$ is $7 \%$. The enhancement of particle numbers in the presence of the instability for $10^{2}<p<10^{3}$ occurs in all the models we considered, but $K$ is reduced only for the pure injection case with $\alpha=0.5$ at quite late times. This implies that a smaller mean diffusion coefficient is not responsible for the shorter acceleration time scale. We believe, instead, that the more efficient acceleration resulted from the larger mean "effective" velocity jump due to the small scale shocks. This also explains the shorter diffusion length as mentioned before. These effects increase as the particle energy increases, because more scatterings are required to reach higher energies. We can see the effects of the finite grid size and no diffusive flux boundary condition for particles at $p \sim 10^{4}$ in the unperturbed cases with momentum dependent diffusion, as discussed above. But this has no effect on the dynamics of the shock or the energy density of the CR, because the distribution function at this energy is very small.

\section{CONCLUSION}

There are two competing consequences of cosmic-ray diffusion on short-wavelength compressional disturbances: (1) CR diffusion provides friction against compression and decompression (Ptuskin's damping). (2) The large-scale gradient of CR pressure behaves nearly as a constant volume force which 

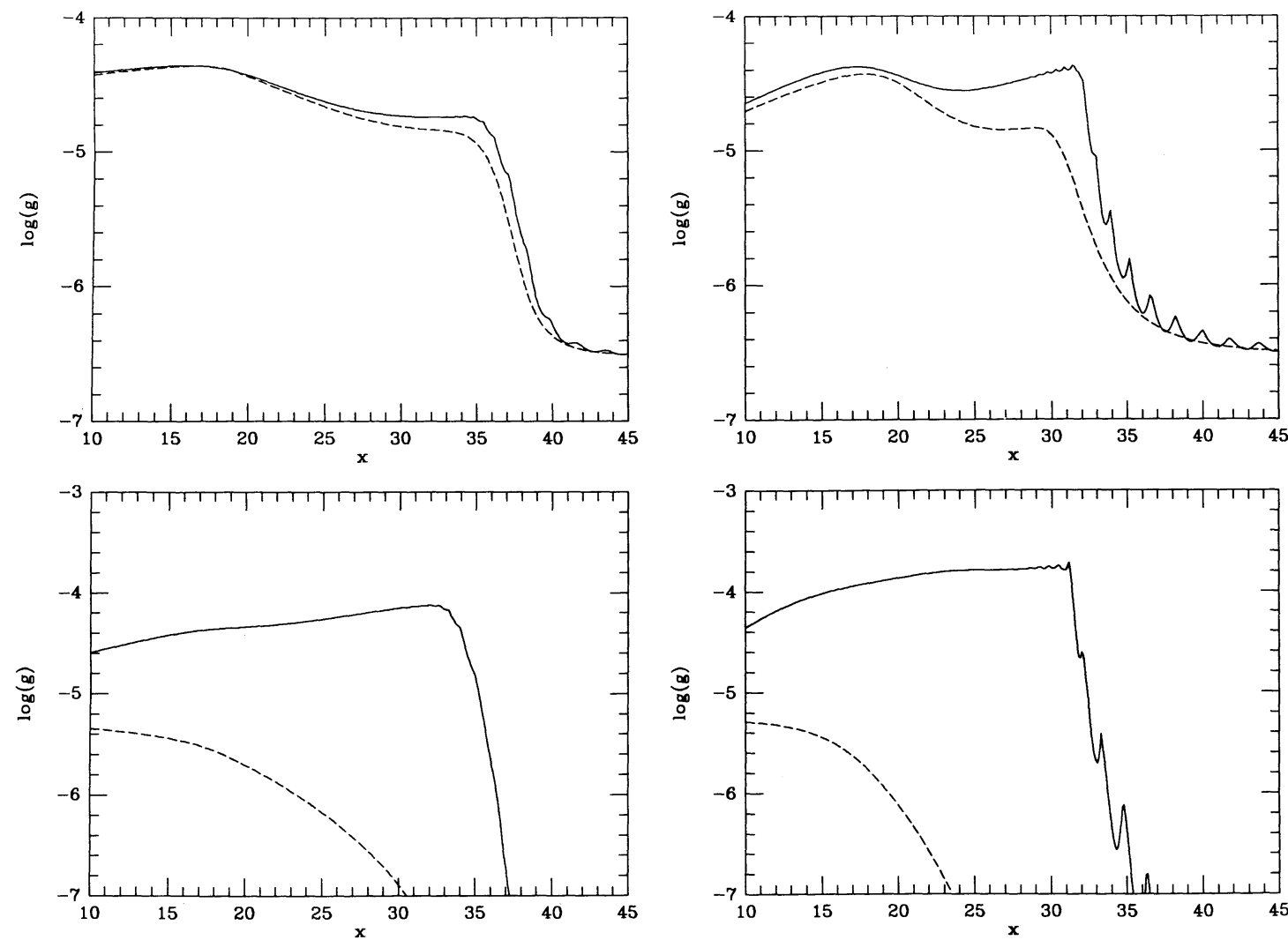

Fig. 5.-Particle distribution function $g=f p^{4}$ for $p=7.3 \times 10^{-3}$ at $t=150$, accelerated by the piston driven shocks. The preexisting particle cases with $\langle\kappa\rangle=p^{0.25}$ (model P2: upper left panel) and $\langle\kappa\rangle=p^{0.5}$ (model P3: upper right panel), and the pure-injection cases with $\langle\kappa\rangle=p^{0.25}$ (model I2: lower left panel) and $\langle\kappa\rangle=p^{0.5}$ (model I3: lower right panel) are shown. The solid lines are the cases perturbed by sound waves of $\lambda=0.2$ and $A=0.2$, while the dashed lines are unperturbed case.

can amplify sound waves propagating in the direction of increasing $P_{c}$ (Drury's instability). If the driving force for Drury's instability dominates Ptuskin's damping, the amplitudes of gas sound waves will grow. One should note that this is a propagating instability as opposed to a standing instability such as the Rayleigh-Taylor instability. In one dimensional geometry, for example, if a small arbitrary perturbation is created in the unstable region, then two sound waves, one propagating to the left and one to the right, will be generated. The wave traveling in the direction to which $P_{c}$ increases will be amplified, while that traveling in the opposite direction will be damped out.

We have shown that due to Drury's instability gas sound waves can steepen into small-scale shocks in the precursor region of strong CR modified shocks. As a result, particles can be more quickly accelerated to the momenta corresponding to the small-scale shock velocities, enhancing the particle distribution there. Also particles can be injected at the small-scale shocks even after the large-scale gas subshock disappears. However, the instability does not significantly change the overall cosmic-ray energy density and the acceleration efficiency. This is because the instability condition (see eq. [3.1]) guarantees that the unstable wavelength of the perturbation is much smaller than the diffusion length for sound waves, so the diffusion associated with sound waves effectively smoothes out the spatial perturbation of the CR on the scale under consideration. On the other hand, it may have more interesting effects on astrophysical shocks, because the density and temperature of the gas in the precursors show multiple small scale shocks.

We can suggest one instance where the consequences of Drury's instability might have already been observed in astrophysical shocks. Fiedler et al. (1987) have reported sharp flux variations (with amplitudes up to $100 \%$ and time scales of several weeks) in a few compact extragalactic radio sources that are most easily understood as either scattering or refraction through small-scale, large-amplitude electron density fluctuations in the intervening interstellar medium. They refer to these as ESE, or extreme scattering events. Romani (1988) has suggested that the ESE may be associated with large radio continuum loops generated by old supernova remnants or collective superbubbles (see also Fiedler et al. 1991). The ESE seem to be produced by density features moving at speeds of several times $10 \mathrm{~km} \mathrm{~s}^{-1}$ and having scales of a fraction of an astronomical unit (The observations are not sensitive to other scales, even if they are present). If these features are identified with wave fronts roughly parallel to edge-on shocks (e.g., Fiedler et al. 1991) the electron densities in the features would be $\sim 10^{2} \mathrm{~cm}^{-3}$. It seems plausible that these features could be the result of density fluctuations in the interstellar medium that have been swept into an advancing shock structure and amplified by Drury's instability. Because the CR precursor decelerates gas before it enters the shock (in the rest frame of the shock), such waves would also tend to be refracted into a propagation more nearly parallel to the shock normal. That would 

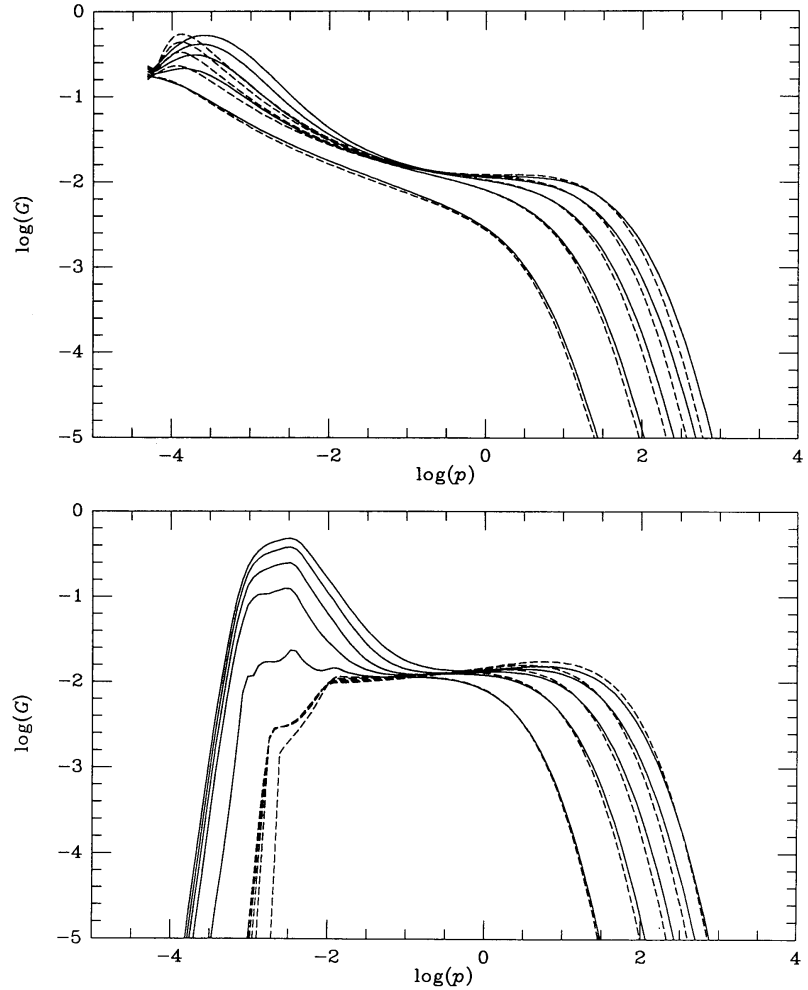

Fig. 6.-Particle distribution function integrated over the spatial grid $(G)$ for the piston driven shocks at $t=30,60,90,120$, and 150 . The model with preexisting particles and $\langle\kappa\rangle=p^{0.5}$ (model P3) is plotted in the top frame, while the model with injected particles and $\langle\kappa\rangle=p^{0.5}$ (model I3) is plotted in the bottom frame. The solid lines are the cases perturbed by sound waves of $\lambda=0.2$ and $A=0.2$, while the dashed lines are unperturbed case.

enhance their ability over randomly oriented density waves to produce ESE. For all this to work one requires a diffusion length large compared to $10^{12} \mathrm{~cm}$, of course. We can see that $x_{d s} \sim 10^{19} \kappa_{25} / u_{s 10} \mathrm{~cm}$, where $\kappa_{25}$ is the diffusion coefficient at the shock in units of $10^{25} \mathrm{~cm}^{2} \mathrm{~s}^{-1}$, and $u_{s 10}$ is the shock speed in units of $10 \mathrm{~km} \mathrm{~s}^{-1}$. Although it is hard to make confident choices for the diffusion coefficient and shock velocity, these values seem reasonable (see, e.g., KJ). Thus, we can conclude that the observed density features are well into the range where Drury's instability can act on them. On the other hand, the scales exceed by several orders of magnitude the Larmour radii for shocked thermal ions, for example, so they should be broad compared to the thickness of the shock itself. Also, the diffusion time scale, $t_{d s} \sim x_{d s} / u_{s}$, should be much less than the lifetime of such shocks, allowing the possibility that the shocks have had time to become mediated by the CR.

It has been suggested that Drury's instability may prohibit the existence of the very efficient CR-dominated strong shocks with highly energetic particles (e.g., $\gamma_{c}=4 / 3$ ) which have been found as one of the multiple solutions in the steady state limit (Baring \& Kirk 1991). Jones \& Ellison (1991) argued that the existence of these efficient shocks is unphysical and resulted from the lack of conservation of particle flux. They also suggested that the escape of the highly energetic particles due to the momentum-dependent diffusion coefficient could eliminate such solutions. One should note, however, that the multiple solutions exist only for the cases of $\gamma_{c} \lesssim 4 / 3$ and the physically meaningful minimum of $\gamma_{c}$ is $4 / 3$. The initial particle distribution with $\gamma_{c} \approx 4 / 3$ has to be accelerated for a very long time before the shock becomes CR-dominated and, hence, unstable against Drury's instability. That is because $\boldsymbol{P}_{c}$ grows linearly as $\gamma_{c}$ decreases to $4 / 3$, while it grows exponentially for $\gamma_{c} \sim 5 / 3$. One can crudely estimate the time scale for the CR shock with $\gamma_{c}=4 / 3$ to reach a steady state is $\gtrsim 1000 t_{d s}$ (Jones \& Kang 1990). If the initial, preexisting particle spectrum is much softer than $p^{-4}$ or if fresh particles are injected from the population of thermal particles, then $\gamma_{c}$ will start from values close to $5 / 3$ and decrease as the particles are accelerated to relativistic momenta, and this will slow down the accleration process. It is unlikely that $\gamma_{c}$ will be spatially constant even if the shock and the particle distribution become steady. Also, except for the special case of a power-law distribution with index $4, \gamma_{c}$ cannot be decreased to all the way to $4 / 3$ unless all the particles become relativistic. But, because the velocity profile in a CRdominated shock is no longer only a step with a simple compression ratio of 4 , the distribution function will generally not be a simple power law. Because of the long times involved, it is not possible for us to carry out such calculations with available resources until a CR-dominated steady shock with $\gamma_{c} \sim 4 / 3$ is reached. So we were not able to test the above suggestion by Jones \& Ellison (1991). We are dubious that such shocks with $\gamma_{c}=4 / 3$ will be important in real physical situation, however, because of all the above issues. But if they exist, it is doubtful
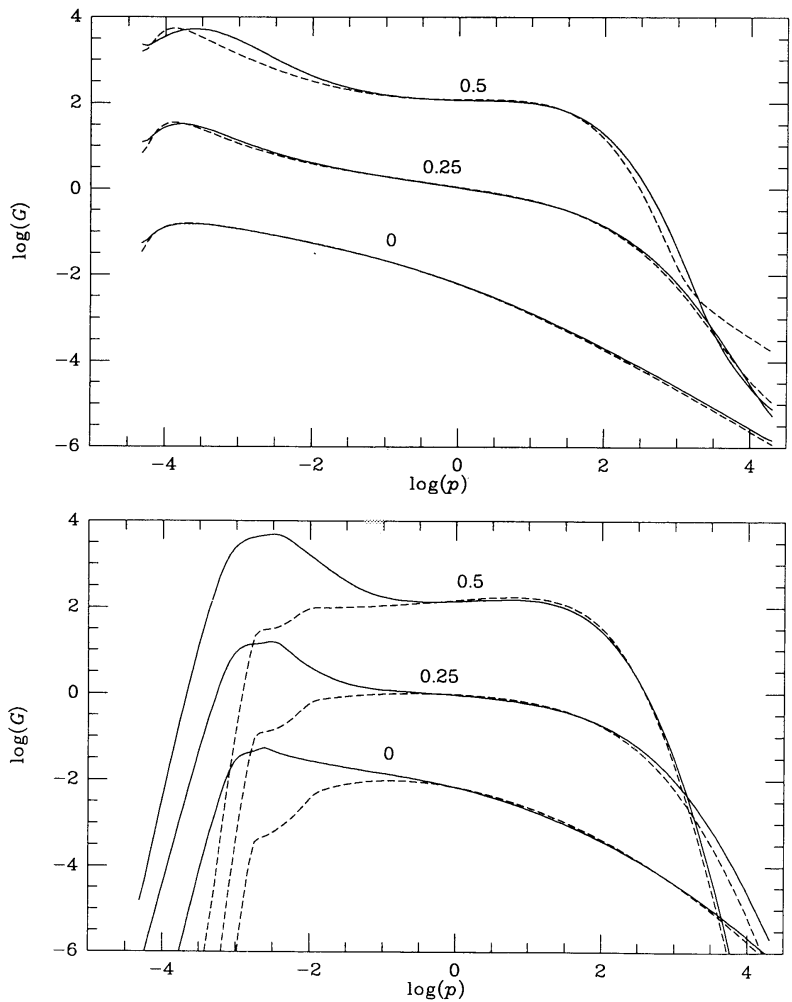

FIG. 7.-Particle distribution function integrated over the grid $(G)$ for the piston driven shocks at $t=150$. The top frame shows the cases with preexisting particles (model P1, P2, and P3), while the bottom frame shows the pure injection cases (model I1, I2, and I3). The solid lines are the cases perturbed by sound waves of $\lambda=0.2$ and $A=0.2$, while the dashed lines are the unperturbed case. The curves are labeled with the value of $\alpha$, where $\kappa=p^{\alpha}$. The values of $\log (G)$ are shifted by 2 for $\alpha=0.25$, and by 4 for $\alpha=0.5$ for clarity. Distribution functions $f$ are expressed in units of the number density $\rho_{N} / m$, where $\rho_{N}$ is used to normalize gas mass density. The momentum $p$ is expressed in units of $m c$. The velocity is normalized so that $u_{p} / c=0.01$. 

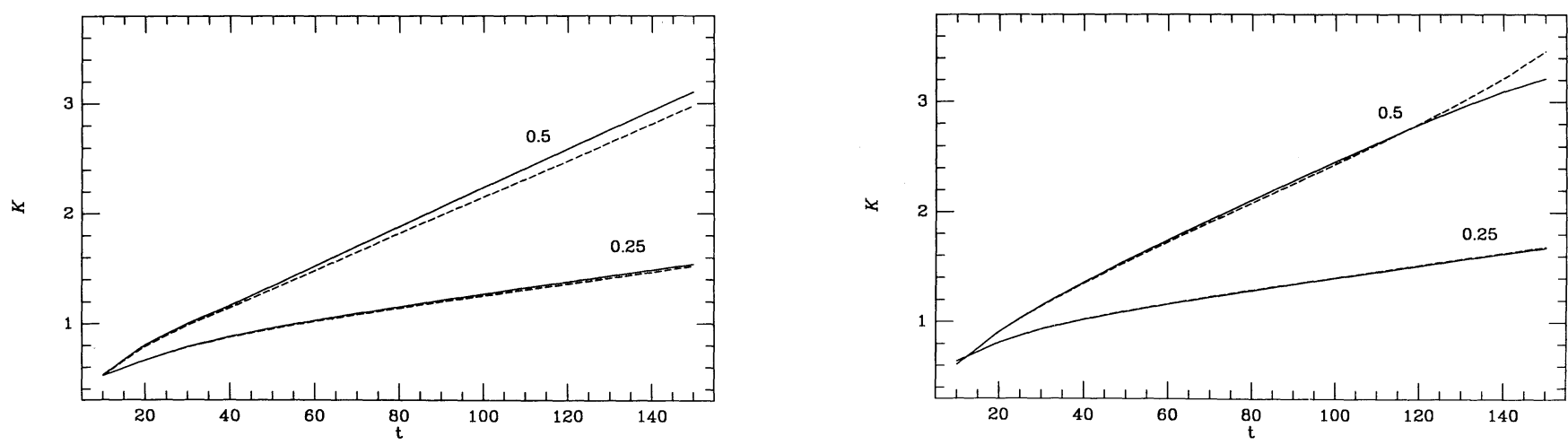

Fig. 8.-Mean diffusion coefficient averaged over the grid $(K)$ against time for the same shocks as in Fig. 7. The cases of $\alpha=0$ for which $K=1$ are omitted. The line types and labels are the same as in Fig. 7.

that Drury's instability will significantly affect the development of and prohibit the existence of CR-dominated shocks with $\gamma_{c} \sim 4 / 3$, because our numerical calculations of CR-dominated shocks with $\gamma_{c}>4 / 3$ show no dramatic changes from unperturbed shocks.

We are very grateful to Paul Woodward for generously making a copy of his PPM hydrodynamics code available to us. Luke Drury suggested that we study this problem using the transport code. We want to express our gratitude to him for the initial suggestion. We are also grateful to Ralph Fiedler for making us aware of the observations of ESE. The work by H. K. and T. W. J. was supported in part by the NSF through grant AST-8720285 and in part by the University of Minnesota Supercomputer Institute. The work by D. R. was in part supported by David and Lucille Packard Foundation Fellowship through Jeremy Goodman at Princeton University. We are also grateful to the MSI and Princeton University for their hospitality while this work was carried out.

\section{APPENDIX A}

\section{NOTE TO NUMERICAL SCHEME}

The details of our numerical schemes can be found in Jones \& Kang (1990) for the two-fluid code and KJ for the transport code. In the previous studies (DF; Falle \& Giddings 1985; Jones \& Kang 1990; KJ) the CR pressure was ignored in solving the Riemann problems by assuming the $P_{c}$ is nearly continuous across each cell boundary. This is a good approximation if the shock structure is well resolved. Since the instability introduces smaller scales than the characteristic scale of the unperturbed shocks (diffusion length of the shock), it will require us to use higher resolution in order to achieve the same level of convergence without perturbations. In the present work we have substantially improved the rate of convergence of the above two codes by including the pressure force due to the CR pressure in the Riemann Solver. We assume that the $\boldsymbol{P}_{c}$ gradient is constant during the time step, so the $\boldsymbol{P}_{c}$ gradient force can be treated as a constant external force,

$$
f_{c}=-\frac{1}{\rho} \nabla P_{c}
$$

Then the left and right gas pressure at each cell boundary used in Riemann problems should be corrected as

$$
\begin{aligned}
& \left(P_{g L}\right)_{\mathrm{corr}}=P_{g L}+\frac{1}{2} C_{s, L} \Delta t\left(f_{c L}+f_{g L}\right), \\
& \left(P_{g R}\right)_{\mathrm{corr}}=P_{g R}-\frac{1}{2} C_{s, R} \Delta t\left(f_{c R}+f_{g R}\right),
\end{aligned}
$$

where $P_{g L}\left(P_{g R}\right), C_{s, L}\left(C_{s, R}\right), f_{c L}\left(f_{c R}\right)$, and $f_{g L}\left(f_{g R}\right)$ are the time-averaged gas pressure, the Lagrangian sound speed, the CR pressure force, and a body force such as gravity, respectively, in the left (right) state to the cell boundary, and $\Delta t$ is the time step.

\section{APPENDIX B}

\section{TEST CALCULATION}

In order to assure that our numerical code can simulate the instability properly, we have calculated eigenwaves in the exponential background described in $\S 5$ with our two-fluid code. We have shown in KJ that our transport code generally gives results consistent with two-fluid calculations. The initial conditions are generated according to the exponential background equations (4.1) and the perturbation equations (4.2). The initial velocity perturbation at $t=0$ is

$$
\tilde{u}=A c_{s 0} \exp (-i k x) \text {, }
$$



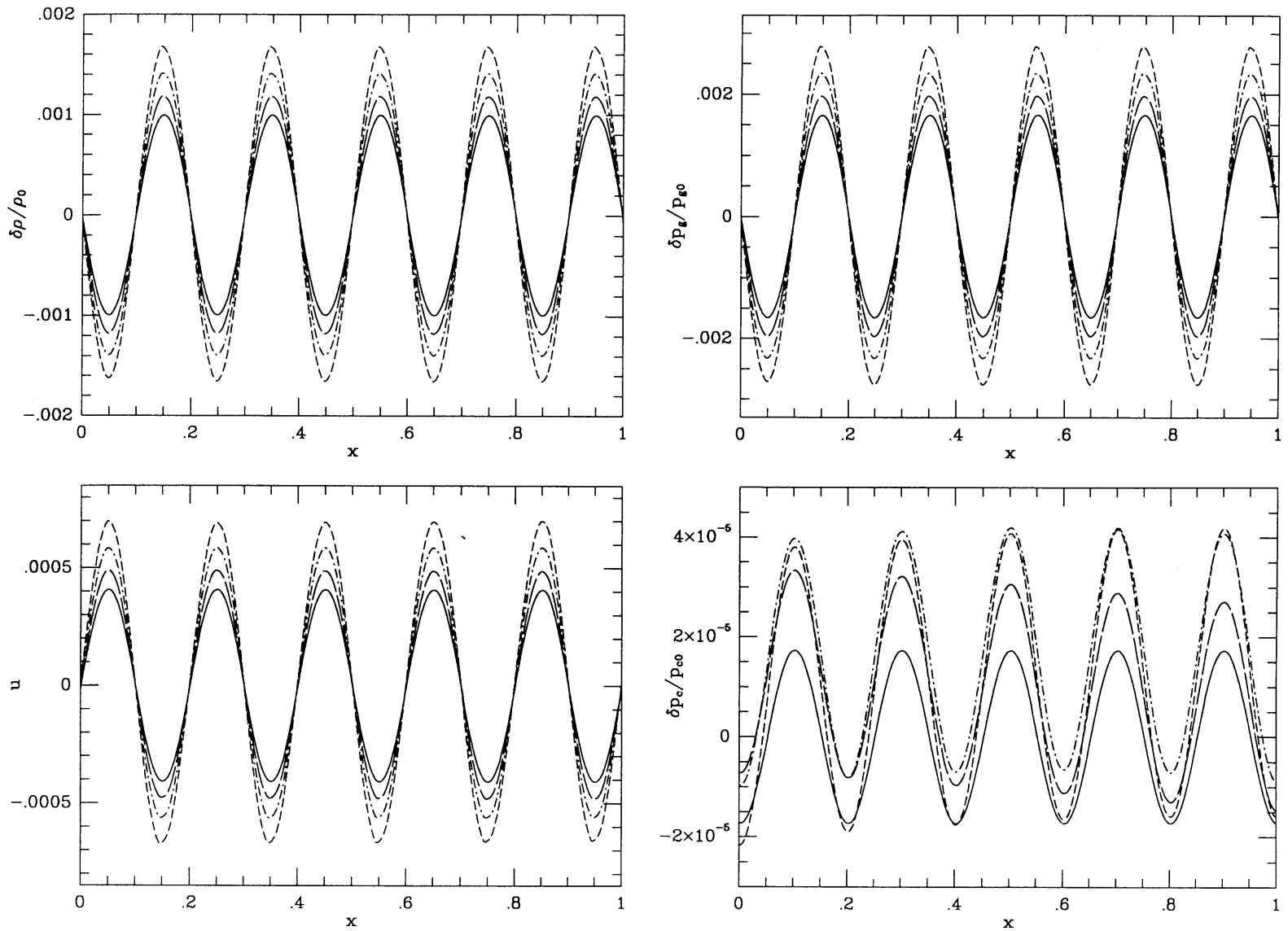

Fig. 9.-Time-dependent growth of eigenwaves in the exponential background described in the text. Normalized perturbed quantities [e.g., $\left.\left(\rho-\rho_{0}\right) / \rho_{0}\right]$ are plotted except for the velocity. The initial amplitude $A$ is 0.001 . The solid lines represent the initial waves at $t=0$, the long-dashed lines at $t=P_{w}$, dot-dashed lines at $t=2 P_{w}$, and dashed lines at $t=3 P_{w}$, where $P_{w}=0.488$ is the wave period.

where $A$ is the normalized amplitude and $k=2 \pi / \lambda$ is the wave number. The pressure force is balanced by a gravity, $f_{g}$,

$$
f_{g}=\frac{P_{g 0}+P_{c 0}}{L_{\rho 0}} .
$$

The diffusion of the CRs is balanced by a CR energy sink, $\Sigma$,

$$
\Sigma=-\frac{\kappa_{0}}{L^{2}\left(\gamma_{c}-1\right)} P_{c 0} \exp \left(\frac{x}{L}\right)
$$

We took $L=-1, \rho_{0}=1, P_{c 0}=1, P_{g 0}=0.1, \kappa_{0}=1$ and $\beta=0.0$. For $\lambda=0.2$, the eigenvalue of the dispersion relation is $w=12.9-0.349 i$ for $\gamma_{c}=4 / 3$. The boundary conditions except for the CR pressure are calculated by extending the exponential distribution for the background and by calculating the perturbation equations (4.2) at the left and right boundaries. This is only approximately true for the CR pressure because of the implicit nature of the finite difference scheme we adopted for that quantity. Figure 9 shows the growth of such eigenwaves. The amplitude of $P_{c}$ perturbations is about two orders of magnitude smaller than that of $P_{g}$ as expected from the linear perturbation analysis. One should notice that eigenwaves for the gas dynamic variables are followed quite accurately, even though the zero point of the $P_{c}$ perturbation is shifted due to accumulated numerical errors. The numerical growth rate of these waves $(\approx 0.35)$ is consistent with the initial eigenvalue $b=0.349$. For the diffusion coefficient inversely proportional to the density $(\beta=-1)$, the initial perturbations are damped out as expected.

\section{REFERENCES}

Baring, M. G., \& Kirk, J. G. 1991, A\&A, in press

Blandford, R. D., \& Eichler, D. 1987, Phys. Rep., 154, 1

Chalov, S. V. 1988, Sov. Astron. Lett., 14, 114

Colella, P., \& Woodward, P. R. 1984, J. Comp. Phys., 54, 174

Dorfi, E. A. 1984, Adv. Space Res., 4, (2-3), 205

1990, A\&A, 234, 419

Dorfi, E. A., \& Drury, L O’C. 1985, Proc. 19th Internat. Cosmic Ray Conf. (La Jolla) 3,121

Drury, L. O'C. 1984, Adv. Space Res., 4, (2-3), 185

Drury, L. O'C., \& Falle, S. A. E. G. 1986, MNRAS, 223, 353 (DF)

Drury, L. O'C., \& Völk, H. J. 1981, ApJ, 248, 344

Falle, S. A. E. G., \& Giddings, J. R. 1987, MNRAS, 225, 399

Fieldler, R. L., Dennison, B., Johnston, K. J., \& Hewish, A. 1987, Nature, 326, 675
Fielder, R. L., Dennison, B., Johnston, K. J., Waltham, E. B., \& Simon, R. S. 1991, preprint

Jones, F. C., \& Ellison, D. C. 1991, Space Sci. Rev., in press

Jones, T. W., \& Kang, H. 1990, ApJ, 363, 499

Kang, H., \& Jones, T. W. 1990, ApJ, 353, 149 1991, MNRAS, 249, 439 (KJ)

Ptsuskin, V. S. 1981, Ap\&SS, 76, 265

Romani, R. W. 1988, in AIP Conf. Proc. 174, Radio Wave Scattering in the Interstellar Medium, ed. J. Cordes, B. Rickett, \& D. Backer (New York: AIP), 156

Völk, H. J., Drury, L. O’C., \& McKenzie, J. F. 1984, A\&A, 130, 19

Zank, G. P., Axford, W. I., \& McKenzie, J. F. 1990, A\&A, 233, 275 